\title{
Quotients of Local Banach Algebras are Local Banach Algebras
}

By

\author{
Lothar M. SCHMITT*
}

\begin{abstract}
We show that quotients of local Banach algebras are local Banach algebras. This solves a problem posed by B. Blackadar in his book on $K$-Theory [BLA1]. Furthermore our techniques allow to prove additional density Theorems for the $K$-Theory of normed algebras stable under inverses and logarithms.
\end{abstract}

\section{Introduction}

Local Banach algebras arise naturally in the context of differential geometry. In commutative differential geometry the algebra of $\mathcal{C}^{\infty}$-functions on a manifold $\mathfrak{M}$ with compact support constitutes the natural local Banach algebra which represents the differentiable structure of $\mathfrak{M}$. In his pioneering work on non-commutative differential geometry and cyclic cohomology A. Connes [CoN 1] has constructed a pairing between the cyclic cohomology of the underlying algebra $\mathcal{A}$ and its $K$-Theory. In many cases $\mathcal{A}$ is a local Banach algebra and the $K$-Theory of $\mathcal{A}$ can identified with the $K$-Theory of its closure. In his book on $K$-Theory B. Blackadar poses the problem whether the quotient of local Banach algebras is a local Banach algebra-cf. [BLA1, p. 19]. In this paper we shall give an affirmative positive solution to this question. Furthermore we can use our technique to identify the $K$-Theory of normed algebras that are closed under inverses and logarithms with the $K$-Theory of their closures. In fact this class is good enough-see Proposition 1.6 and Corollary 2.3.- to permit the holomorphic functional calculus needed to establish Bott periodicity and the well known cyclic sixterm exact sequence of complex

Communicated by H. Araki, May 21, 1990. Revised February 8, 1991. 1991 Mathematics Subject Classification: 46L80, 46H10, 16A54.

* Fachbereich Mathematik/Informatik, Universität Osnabrück, D-4500 Osnabrück, Germany. 
$K$-Theory. Furthermore one can apply the density theorems mentioned above to obtain short proofs of results by A. Connes and $\mathbb{P}$. Jolissaint-see [JoL1]-on the $K$-Theory of spaces of rapidly decreasing functions on certain groups.

\section{Algebras with Open Groun of Imvertibles}

Let $\mathcal{A}$ be an algebra over $\mathbb{C}$. Then we shall write $\mathscr{A}^{0}$ for the unital algebra $\mathscr{A} \oplus \mathbb{C}$ with multiplication $(x, \lambda)(y, \mu)=(x y+\lambda y+\mu x, \lambda \mu), x, y \in \mathcal{A}, \lambda, \mu \in \mathbb{C}$. Let $M_{n}(\mathcal{A}), n \in \mathbb{N}$, denote the $n \times n$ matrices with entries in $\mathcal{A}$. We shall use the notation $x \oplus y, x \in M_{n}(\mathcal{A}), y \in M_{m}(\mathcal{A}), n, m \in \mathbb{N}$ to denote the matrix

$$
\left[\begin{array}{ll}
x & 0 \\
0 & y
\end{array}\right] \text {. }
$$

If $\mathcal{A}$ is unital, then we shall write $\mathrm{GL}_{n}(\mathcal{A})$ for the group of invertible elements in $M_{n}(\mathcal{A})$. If $\mathcal{A}$ is unital and normed, then we shall denote the path component of the identity in $\operatorname{GL}_{n}(\mathcal{A})$ by $\mathrm{GL}_{n}^{0}(\mathcal{A})$. The suspension $\mathcal{S}(\mathcal{A})$ and the cone $\mathcal{C}(\mathcal{A})$ of $\mathcal{A}$ are by definition the following:

$$
\mathcal{C}(\mathcal{A})=\{f:[0,1] \rightarrow \mathcal{A} \mid f \text { is continuos and } f(0)=0\}
$$

and $S(\mathcal{A})=\{f \in \mathcal{C}(\mathcal{A}) \mid f(1)=0\}$.

1.11. Lemmma. Let $\mathcal{A}$ and $\mathscr{B}$ be unital algebras, and let $\varphi: \mathcal{A} \rightarrow \mathscr{B}$ be a surjective homomorphism. If $u \in \mathscr{B}$ is invertible, then there exists $V \in \mathbb{G L}_{2}(\mathcal{A})$ such that $\varphi(V)=u \oplus u^{-1}$. If $\mathcal{A}$ and $\mathscr{B}$ are normed algebras, then $u \oplus u^{-1} \in$ $\mathrm{GL}_{2}^{0}(\mathscr{B})$ and $V$ can be chosen in $\mathrm{GL}_{2}^{0}(\mathcal{A})$.

Proof. This can be obtained immediately from the formular given on the bottom of page 108 in [KAR1].

The next Proposition generalizes [BLA1, Proposition 3.4.6]. As mentioned there it may be known.

1.2. Proposition. Suppose that $\mathcal{A}$ is a normed algebra and $\mathcal{G}$ is a closed, two-sided ideal in $\mathcal{A}$. Then one has:

a) The homomorphism $\Phi: S(\mathcal{A}) / \mathcal{S}(\mathcal{G}) \rightarrow S(\mathcal{A} / \mathcal{G})$ given by

$$
f+\mathcal{S}(\mathcal{G}) \mapsto \pi \circ f
$$

is one-to-one and has dense image in $S(\mathcal{A} / \mathcal{G})$. Here $\pi$ stands for the quotient map $\mathcal{A} \rightarrow \mathcal{A} / \mathcal{G}$. 
b) If $\mathcal{A}$ is a Banach algebra, then $\Phi$ is surjective.

c) Let $\mathcal{A}$ be a Banach algebra, $f:[0,1] \rightarrow \mathcal{A} / \mathcal{G}$ be a norm-continuous path and $X_{0} X_{1} \in \mathcal{A}$ be such that $\pi\left(X_{k}\right)=f(k), k=0,1$. Then there exists a norm-continuous path $F:[0,1] \rightarrow \mathcal{A}$ satisfying $F(k)=X_{k}, k=0,1$ and $\pi(F(t))=f(t), t \in[0,1]$.

Proof. a) It is clear that $\Phi$ is a well defined homomorphism and oneto-one. If $f \in \mathcal{S}(\mathcal{A} / \mathcal{G})$, then by the uniform continuity of $f$ it can be approximated by a piecewise linear function $g \in S(\mathcal{A} / \mathcal{G})$. $g$ can be lifted to $S(\mathcal{A})$. To prove b) check that for a piecewise linear lift $G \in S(\mathcal{A})$ of $g \in S(\mathcal{A} ! \mathcal{G})$ one has that $\|\Pi(G)\| \leqslant\|g\|$, where $\Pi: S(\mathcal{A}) \rightarrow \mathcal{S}(\mathcal{A}) / \mathcal{S}(\mathcal{G})$ is the quotient map. For the proof of c) see [BLA1, Proposition 3.4.6].

1.3. Defimition. Let $\mathcal{A}$ be a normed algebra with the following property: If $x \in M_{n}\left(\mathcal{A}^{0}\right), n \in \mathbb{N}, U=U(0, r) \subset \mathbb{C}$ is an open disk around 0 with radius $r$ greater than $\|x\|$, and $f: U \rightarrow \mathbb{C}$ is a holomorphic function, then $f(x) \in M_{n}\left(\mathscr{A}^{0}\right)$. In this situation we shall say that $\mathcal{A}$ is stable under calculus of power series.

1.4. Remarks. a) Local Banach algebras are of course stable under calculus of power series.

b) Let $\mathcal{A}$ be a normed algebra which is stable under calculus of power series, and let $\mathcal{G}$ be a closed, two-sided ideal in $\mathcal{A}$. Then $\mathscr{A} / \mathcal{G}$ is stable under calculus of power series.

c) Let $\mathcal{A}$ be a unital, normed algebra which is stable under calculus of power series. If $x \in \mathcal{A}$ such that $\|x-\mathbb{1}\|<1$, then $x^{-1}, \log (x) \in \mathcal{A}$. In particular $\operatorname{GL}(\mathcal{A})$ is open in $\mathcal{A}$.

The proof of the following Lemma is left to the reader-compare [BLA1].

1.5. Lemma. For any unital, normed algebra $A$ the following statements hold:

a) $\operatorname{GL}(\mathcal{A})=\operatorname{GL}(\overline{\mathcal{A}}) \cap \mathcal{A}$ if and only if $\mathrm{GL}(\mathcal{A})$ is open in $\mathcal{A}$, where $\overline{\mathcal{A}}$ denotes the completion of $\mathcal{A}$.

b) If $\mathrm{GL}(\mathcal{A})$ is open in $\mathcal{A}$, and $p$ and $q$ are idempotents in $\mathcal{A}$, then one has:

i) Up to the fact that one possibly has to consider $p \oplus 0$ and $q \oplus 0$ in $M_{4}(\mathcal{A})$ the notions of homotopy, similarity and algebraic equivalence coincide for $p$ and $q$.

ii) If $\|p-q\|<\| 2 p-\mathbb{1}^{-1}$, then $p$ and $q$ are homotopic.

c) If $\mathrm{GL}(\mathcal{A})$ is open in $\mathcal{A}$, and $\mathcal{G}$ is a closed, two-sided ideal in $\mathcal{A}$, then $\operatorname{GL}(\mathcal{A} / \mathcal{G})$ is open in $\mathcal{A} / \mathcal{G}$. 
1.6. Proposition. Let $\mathcal{A}$ and $\mathscr{B}$ be unital, normed algebras with open groups of invertible elements, and let $\varphi: \mathcal{A} \rightarrow \mathscr{B}$ be a continuous, surjective homomorphism. Then $\varphi\left(\mathrm{GL}^{0}(\mathscr{A})\right)=\mathrm{GL}^{0}(\mathscr{B})$.

Proof. Let $\mathcal{G}=\operatorname{ker}(\varphi), \pi: \mathcal{A} \rightarrow \mathcal{A} / \mathcal{G}$ be the quotient map, and let $\Phi: \mathcal{A} / \mathcal{G} \rightarrow \mathscr{B}$ be the bijective homomorphism such that $\varphi=\Phi \circ \pi$. If $u \in \mathrm{GL}^{0}(\mathscr{B})$, then there exists a piecewise linear path $f:[0,1] \rightarrow \mathrm{GL}^{0}(\mathscr{B})$ such that $f(0)=1$ and $f(1)=u$. The path $\Phi^{-1} \circ f$ is continuous. Hence we may suppose $\mathscr{B}=\mathcal{A} / \mathcal{G}$ and $\varphi=\pi$. Let $f:[0,1] \rightarrow \mathrm{GL}^{0}(\mathscr{B})$ be a piecewise linear path such that $f(0)=\mathbb{R}$. Then there exist $t_{0}=0<t_{1}<\cdots<t_{n}=1$ (including all the vertices of $f$ ) such that

$$
\left\|1-f\left(t_{m}\right)^{-1} f\left(t_{m+1}\right)\right\|<1,0 \leqslant m \leqslant n-1 .
$$

Let $b_{m} \in \mathcal{A}, 0 \leqslant m \leqslant n$, be a lift of $f\left(t_{m}\right)^{-1} f\left(t_{m+1}\right)$ with $\left\|\mathbb{1}-b_{m}\right\|<1$. Set $a_{0}=\mathbb{1}$ and $a_{m+1}=a_{m} b_{m}, 0 \leqslant m \leqslant n-1$. Then one has $\left\|1-a_{m}^{-1} a_{m+1}\right\|<1$. The piecewise linear path with vertices $a_{0}, a_{1}, \cdots, a_{n}$ is a lift of $f$ within $\operatorname{GL}(\mathcal{A})$.

\section{Quotients of Local Banach Algebras}

The following Theorem shows that quotients of local Banach algebras are local Banach algebras.

2.1. Theorem. Let $\mathcal{A}$ be a normed algebra which is stable under calculus of power series. Then $\mathcal{A}$ is a local Banach algebra.

Proof. Let $x \in \mathcal{A}$, let $U$ be an open neighborhood of $\sigma(x)$, and let $\gamma$ be a piecewise linear path that surrounds $\sigma(x)$ in $U$. Let $f$ be a function which is holomorphic on $U$. Let $g(\lambda)=(\lambda \mathbb{1}-x)^{-1}, \lambda \in \mathbb{C} \backslash \sigma(x)$. There exists $\delta>0$ such that the following statements are true:

a) for $\lambda, \eta \in \Gamma$ satisfying $|\lambda-\eta|<\delta$ one has: $\left\|\mathbb{1}-g(\lambda)^{-1} g(\eta)\right\|<1$, and

b) for $\lambda \in \Gamma f$ has a power series expansion around $\lambda$ on the disk of radius $\delta$. Now consider a line segment $r \subset \Gamma$ with one endpoint equal to $\lambda$ and length less than $\delta$. Then one has

$$
\int_{\gamma} f(\eta) g(\lambda)^{-1} g(\eta) d \eta=\int_{\gamma} f(\eta) \sum_{\nu=0}^{\infty} g(\lambda)^{\nu}(\lambda-\eta)^{\nu} d \eta
$$

Now one uses the power series for $f$ around $\lambda$ to get a power series in $g(\lambda)$ and $(\lambda-\eta)$ which can be integrated term by term.

2.2. Definition. Suppose that $\mathcal{A}$ is a normed algebra. We shall say that 
$\mathcal{A}$ is stable under inverses and logarithms, if for every $x \in M_{n}\left(\mathcal{A}^{0}\right), n \in \boldsymbol{N}$, with $\|1-x\|<1$ one has $x^{-1}, \log (x) \in M_{n}\left(\mathscr{\ell}^{0}\right)$.

The proof of Theorem 2.1 shows that the following is true:

2.3. Corollary. Suppose that $\mathcal{A}$ is a unital, normed algebra which is stable under inverses and logarithms.

a) If $x \in \mathcal{A}$ and $\sigma(x)=\bigcup_{\nu=1}^{n} \sigma_{\nu}, n \in N$, where the $\sigma_{\nu}$ are pairwise disjoint and compact, then the spectral idempotents associated with the $\sigma_{\nu}$ are elements of $\mathcal{A}$.

b) If $\varepsilon>0$ and $p$ is a idempotent in $\overline{\mathcal{A}}$, then there exists an idempotent, $q \in \mathcal{A}$ satisfying $\|p-q\|<\varepsilon$.

\section{Applications to $K$-Theory}

If $\mathcal{A}$ is an algebra, then let $M_{\infty}(\mathcal{A})$ be the algebraic direct limit of $M_{n}(\mathcal{A})$, $n \in \boldsymbol{N}$, under the embeddings $x \mapsto x \oplus 0, x \in M_{n}(\mathcal{A}), 0 \in \mathcal{A}$. Let $V(\mathcal{A})$ be the set of equivalence classes of idempotents in $M_{\infty}(\mathcal{A})$ with respect to algebraic equivalence. If $[p],[q] \in V(\mathcal{A}), p \in M_{n}(\mathcal{A}), q \in M_{m}(\mathcal{A})$, then define

$$
[p]+[q]=[p \oplus q]
$$

where [•] denotes equivalence classes. This turns $V(\mathcal{A})$ into an abelian semigroup with zero element [0].

3.1. Definition. If $\mathcal{A}$ is a unital algebra, then let $K_{0}(\mathcal{A})$ be the Grothendieck group of $V(\mathscr{A})$. If $\mathcal{A}$ is any algebra, then consider the natural homomorphism

$$
\alpha_{\mathcal{A}}: \mathcal{A}^{0} \rightarrow \boldsymbol{C},(x, \lambda) \mapsto \lambda, x \in \mathcal{A}, \lambda \in \boldsymbol{C} .
$$

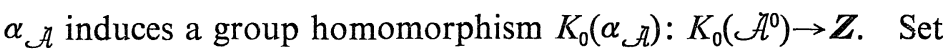

$$
K_{0}(\mathcal{A})=\operatorname{ker}\left(K_{0}(\alpha \mathcal{A})\right) .
$$

With respect to the next two results the reader should compare the density theorems in [AT12] and [KAR1].

3.2. Proposition. Let $\mathcal{A}$ be a normed algebra which is stable under inverses and logarithms. Then the inclusion $i: \mathcal{A} \rightarrow \mathcal{A}$ induces an isomorphism

$$
K_{0}(i): K_{0}(\mathcal{A}) \rightarrow K_{0}(\overline{\mathcal{A}}) .
$$

Proof. Assume w.l.o.g. that $\mathcal{A}$ is unital. If $p$ and $q$ are projections in $\mathcal{A}$ with $p=u q u^{-1}, u \in \operatorname{GL}(\bar{A})$, then one approximates $u$ with $v \in \operatorname{GL}(\mathcal{A})$. If 
$v q v^{-1}$ is sufficently close to $p$, then $v q v^{-1}$ and $p$ are homotopic. Hence all three projections are equivalent, and $K_{0}(i)$ is one-to-one. Corollary 2.3 b) shows that $K_{0}(i)$ is onto.

3.3. Definnition. If $\mathcal{A}$ is a unital, normed algebra, then let $K_{1}(\mathcal{A})$ be the inductive limit of the groups $L_{n}(\mathcal{A})=\mathrm{GL}_{n}(\mathcal{A}) / \mathrm{GL}_{n}^{0}(\mathcal{A}), n \in \mathbb{N}$, where the maps $L_{n}(\mathscr{A}) \rightarrow L_{n+1}(\mathscr{A})$ are induced by the maps

$$
x \mapsto x \oplus \mathbb{1}, x \in M_{n}(\mathcal{A}), \mathbb{1} \in \mathcal{A} .
$$

If $\mathcal{A}$ is any normed algebra, then one defines $K_{1}(\mathcal{A})=K_{1}\left(\mathcal{A}^{0}\right) . \quad K_{1}(\mathcal{A})$ is an abelian group, if the addition it defined as

$$
[u]+[v]=[u \oplus v], u \in \mathbb{G L}_{n}(\mathcal{A}), v \in \mathbb{G L}_{m}(\mathcal{A}), n, m \in \mathbb{N} .
$$

3.4. Proposition. Let $\mathcal{A}$ be a normed algebra such that $\mathrm{GL}_{n}\left(\mathcal{A}^{0}\right)$ is open in $M_{n}\left(\mathcal{A}^{0}\right)$ for all $n \in \mathbb{N}$. Then the inclusion $i: \mathcal{A} \rightarrow \overline{\mathcal{A}}$ induces an isomorphism

$$
K_{1}(i): K_{1}(\mathscr{A}) \rightarrow K_{1}(\overline{\mathcal{A}}) \text {. }
$$

Proof. Suppose w.1.o.g. that $\mathcal{A}$ is unital. Let $u, v \in G L(\mathcal{A})$, and let $f$ be a continuous map $[0,1] \rightarrow G \mathbb{L}(\bar{\AA})$ with $f(0)=u$ and $f(1)=v$. Then $f$ can be replaced by a piecewise linear path $g:[0,1] \rightarrow G \mathbb{L}(\bar{\AA})$ with $g(0)=u$ and $g(1)=v$. Furthermore close to any element in $\operatorname{GL}(\bar{A})$ lies an element of $\operatorname{GL}(\mathcal{A})$ which is in the same path component. Hence $L_{1}(\mathcal{A})=L_{1}(\bar{A})$.

3.5. Remark. B. Blackadar [BLA1] developes $K$-Theory within the class of local Banach algebras. A check through the proofs shows the following: With the help of Lemma 1.1 and the fact that $K_{0}$ is a functor one gets [BLA1, Thm. 5.6.1] for arbitrary algebras. Next one can define the index map as in [BLA1, Def. 8.3.1] and obtaines [Bla1, Prop. 8.3.3 and Prop. 8.3.4] within the class of algebras with open groups of invertibles using Proposition 1.6. [BLA1, Thm. 8.2.2] can be obtained from this, if one considers the exact sequence $0 \rightarrow$ $\mathcal{S}(\mathcal{A}) \rightarrow \mathcal{C}(\mathcal{A}) \rightarrow \mathcal{A} \rightarrow 0$. Furthermore with the help of Proposition 1.6 one can prove [BLA1, Cor. 8.2.3] directly. Using Proposition 1.2 this yields the long exact sequence of $K$-Theory. To get Bott periodicity one uses Corollary 2.3 and the proof given in [BLA1, 9.2]. One has to give a detailed argument for the injectivity of the Bott map as in [PAs1].

3.6. Corollingy. Let $\mathcal{A}$ be a normed algebra such that $\mathrm{GL}_{n}\left(\mathcal{A}^{0}\right)$ is open in $M_{n}\left(\mathcal{A}^{0}\right)$ for all $n \in \mathbb{N}$. Then one has $K_{0}(S(\mathcal{A}))=K_{0}(\mathcal{S}(\overline{\mathcal{A}})$.

3.7. Remark. In [JoL1] P. Jolissaint identifies the $K$-Theory of spaces of 
rapidly decreasing functions on certain discrete groups with the $K$-Theory of the reduced group $C^{*}$-algebra. One can use Corollary 2.3 to shorten the argument. In fact in each case the first step of the given proofs shows that the algebras considered there are stable under inverses and logarithms.

The author is grateful to the referee for a simplified argument in the proof of Proposition 1.6.

\section{References}

[ATr 1] Atiyah, M. Bott periodicity and the index of elliptic operators, Quart. J. Math. Oxford Ser., 19 (1968), 113-140.

[ATr 2] — $\quad K$-Theory, Benjamin, 1967.

[BLA 1] Blackadar, B., K-Theory for Operator Algebras, Mathematical Sciences Research Institute Publications, Springer-Verlag, 1986.

[Con1] Connes, A., Non-Commutative Differential Geometry, Chapter II: The Chern Character in $K$-Homology, Chapter II: De Rham Homology and Non-Commutative Algebra, Publ. Math. I.H.E.S., 62 (1986), 257-360.

[Jol 1] Jolissaint, P., $K$-Theory of reduced $C^{*}$-algebras and rapidly decreasing functions on groups, $K$-Theory, 2 (1989), 723-735.

[KAR1] Karoubi, M., K-Theory. An Introduction, Grundlehren der mathematischen Wissenschaften 226, Springer-Verlag, 1978.

[Pas 1] Pasclike, W., Lectures on $K$-theory for Banach algebras, Notes taken by B. Legan, University of Kansas (1987).

[TAY 1] Taylor, J., Banach algebras and topology, Algebras in Analysis, Editor J.H. Williamson, Academic Press, 1975. 
